\title{
Pulse Construction in OFDM Systems via convex optimization
}

\author{
Jiadong $\mathrm{Xu}$ and Thomas Strohmer \\ Department of Mathematics \\ University of California, Davis \\ Email: jiadong@math.ucdavis.edu,strohmer@math.ucavis.edu
}

\begin{abstract}
In order to reduce the inter-carrier interference (ICI) produced by frequency offset in OFDM systems, we set up an optimization problem to find the transmission pulse which maximizes the signal-to-average-ICI power ratio. Furthermore, by solving a constrained optimization problem, our pulses can satisfy various decay requirements, for instance with respect to a prescribed spectral mask. The latter is an important feature in order to comply with industry standard specifications. Simulation results show that our pulses outperform many currently known transmission pulses with respect to the ICI criterion.
\end{abstract}

Keywords Frequency offset, inter-carrier interference (ICI), pulse-shaping, Orthogonal Frequency Division Multiplexing (OFDM), Optimal pulse.

\section{INTRODUCTION}

OFDM has attracted considerable attention in recent years due to its desirable properties such as its high data rate transmission capability, as well as its robustness to multipath delay spread. On the other hand, OFDM is sensitive to carrier frequency offset, caused by misalignment in carrier frequencies or Doppler shift. This can produce ICI and thus can degrade the overall performance [1].

Many OFDM pulses were designed recently to reduce the ICI effect. Generalized raised-cosine (RC) pulses were proposed in [2]. In [3], the so-called "better than raised cosine" (BTRC) pulse was presented which outperforms the raised-cosine pulse in terms of sensitivity to timing errors. In [4], a family of intersymbol-interference-free polynomial pulses (POLY) was designed, whose Fourier transform can provide an asymptotic decay rate (ADR) of the order $\omega^{-k}$ for any integer $k$, and which show smaller ICI when compared with the aforementioned pulses [5]. A different approach to OFDM pulse shape design, based on time-frequency analysis, can be found in [6], [7], [8].

Our goal in this paper is twofold. Firstly, we want to design OFDM pulses that improve upon existing OFDM pulses with respect to ICI robustness. Secondly, we want to design the pulses such that they also satisfy prescribed spectral decay requirements such as dictated by, e.g., IEEE 802-standard specifications. This additional aspect is very important in practice. Hence, unlike the aforementioned methods which

This work was partially supported by NSF DMS grant 0511461. 
construct "nice" time-limited pulses directly, in this paper we propose a pulse design method which maximizes the total signal-to-average-ICI power ratio (SIR) with respect to user-defi ned spectral decay conditions by solving a constrained convex optimization problem. By doing so we obtain an OFDM pulse that achieves the largest SIR - which in turn leads to the smallest ICI effect - under the given spectral decay requirements. We also provide some details concerning the numerical implementation of our method.

\section{PROBlem Description}

We assume that the reader is familiar with the basics of OFDM (cf. e.g. [1]) and present only those details necessary to clarify notation and to formulate the problem under consideration. We follow mainly the setup and notation in [3].

The OFDM transmission signal at time $t$ is given by

$$
x(t)=e^{i 2 \pi f_{c} t} \sum_{k=0}^{N-1} a_{k} p(t) e^{i 2 \pi f_{k} t},
$$

where $i=\sqrt{-1}, f_{c}$ is the carrier frequency, $N$ is the number of subcarriers, $a_{k}$ is an OFDM constellation point, $f_{k}$ is the subcarrier frequency of the $k$-th subcarrier and $p(t)$ is the time limited pulse which we need to design. Furthermore, we assume that $a_{k}$ is an i.i.d. random variable with mean zero and variance $\frac{N_{0}}{2}$. We also have

$$
f_{k}-f_{m}=\frac{k-m}{T}
$$

to ensure subcarrier orthogonality, which is

$$
\int_{-\infty}^{\infty} p(t) e^{i 2 \pi\left(f_{k}-f_{m}\right) t} d t= \begin{cases}1 & k=m \\ 0 & k \neq m\end{cases}
$$

for $k, m=0,1, \ldots, N-1$, where $\frac{1}{T}$ is the subcarrier frequency space, and $T$ is the symbol period. In the presence of frequency offset $\Delta f$ between receiver and transmitter, after multiplication by $e^{-i 2 \pi\left(f_{c}-\Delta f\right) t}$, the received signal is

$$
r(t)=e^{i 2 \pi \Delta f t} \sum_{k=o}^{N-1} a_{k} p(t) e^{i 2 \pi f_{k} t} .
$$

We obtain the decision variable for the $m$-th subcarrier

$$
\hat{a}_{m}=a_{m} P(-\Delta f)+\sum_{k=0, k \neq m}^{N-1} a_{k} P\left(\frac{m-k}{T}-\Delta f\right),
$$

where $P(w)$ is the Fourier Transform of $p(t)$ and the second term in the right hand side is ICI produced by frequency offset. After taking expectation, we get the average ICI power for the $m$-th symbol

$$
\bar{\delta}_{I C I}^{m}=\sum_{k=0, k \neq m}^{N-1}\left|P\left(\frac{k-m}{T}+\Delta f\right)\right|^{2}
$$


as (13) in [3]. Furthermore, we take the same defi nition in [3] as the average signal power to average ICI power ratio (SIR) for the $m$-th symbol

$$
\operatorname{SIR}^{m}=\frac{|P(\Delta f)|^{2}}{\bar{\delta}_{I C I}^{m}}
$$

Since the defi nitions in (6) and (7) depend on the index $m$, we defi ne the total average ICI power (TICI) for a pulse in an $N$-subcarrier OFDM system as

$$
\mathrm{TICI}=\sum_{m=0}^{N-1} \bar{\delta}_{\mathrm{ICI}}^{m}=\sum_{k=-(N-1), k \neq 0}^{N-1}(N-|k|)\left|P\left(\frac{k}{T}+\Delta f\right)\right|^{2},
$$

and the total average signal power to average ICI power (TSIR) for a pulse in an N-subcarrier OFDM system as

$$
\mathrm{TSIR}=\frac{N|P(\Delta f)|^{2}}{\sum_{m=0}^{N-1} \bar{\delta}_{\mathrm{ICI}}^{m}}
$$

which are independent of index $m$. For a OFDM system with $\mathrm{N}$ subcarriers, $\bar{\delta}_{I C I}^{m}$ changes with index $m$ while TICI is fixed, which can reflect the ICI power of the whole OFDM symbol rather than symbol by symbol. We know that large TICI (or small TSIR respectively) implies high distortion in OFDM systems. Thus in the next section, we will minimize TICI to achieve an optimal time limited pulse which satisfi es (3).

\section{OFDM PULSE DESIGN VIA CONSTRAINED OPTIMIZATION}

Consider a time limited pulse $p$ which satisfi es (3) with $p(t)=0$ when $|t| \geq \frac{T(1+\alpha)}{2}$. Since it is a $L^{2}$ function in the domain $\left[-\frac{T(1+\alpha)}{2}, \frac{T(1+\alpha)}{2}\right]$, from Fourier analysis [9], such a function can be expressed as

$$
p(t)=\left\{\begin{array}{cc}
\sum_{-\infty}^{\infty} \frac{c_{j}}{(1+\alpha) T} e^{\frac{2 \pi i t j}{(1+\alpha) T}} & t \in\left[\frac{(-1-\alpha) T}{2}, \frac{(1+\alpha) T}{2}\right] \\
0 & t \notin\left[\frac{(-1-\alpha) T}{2}, \frac{(1+\alpha) T}{2}\right] .
\end{array}\right.
$$

Thus the Fourier Transform of $p$ is given by

$$
P(w)=\sum_{j=-\infty}^{\infty} c_{j} \frac{\sin \left(\pi T(1+\alpha)\left(w-\frac{j}{(1+\alpha) T}\right)\right)}{(1+\alpha) T \pi w-\pi j} .
$$

We note that a time-limited pulse (intersymbol-interference-free or not) can at best have an ADR in the Fourier domain of the order $e^{-|\omega|^{\alpha}}$ with $\alpha<1$, this follows from [10].

Without loss of generality we let $T=1$ in the rest of the paper. Using Taylor expansion, we have

$$
P(k+\Delta f)=P(k)+P^{\prime}(k) \Delta f+\mathcal{O}\left(|\Delta f|^{2}\right)=P^{\prime}(k) \Delta f+\mathcal{O}\left(|\Delta f|^{2}\right),
$$

thus

$$
\mathrm{TICI}=\sum_{k=-(N-1), k \neq 0}^{N-1}(N-|k|)|P(k+\Delta f)|^{2}=\sum_{k=-(N-1), k \neq 0}^{N-1}(N-|k|)\left|P^{\prime}(k)\right|^{2}|\Delta f|^{2}+\mathcal{O}\left(|\Delta f|^{3}\right) .
$$


For small $|\Delta f|$ the dominant part of TICI is the fi rst term. Hence instead of maximizing TICI directly, we try to maximize the dominant term, which leads to minimize $\sum_{k=-(N-1), k \neq 0}^{N-1}(N-|k|)\left|P^{\prime}(k)\right|^{2}$.

We now proceed by setting up the optimization problem with respect to the dominant TICI term. We let the $2 N-1$ vector $b$ have components $b(0)=1$ and $b(k)=0$ for $k \in\{-(N-1), \ldots, N-1\}$, and defi ne the function $g(w, j)=\frac{\sin \left(\pi(1+\alpha)\left(w-\frac{j}{1+\alpha}\right)\right)}{(1+\alpha) \pi w-\pi j}$. Then from (11) we have

$$
\sum_{k=-(N-1), k \neq 0}^{N-1}(N-|k|)\left|P^{\prime}(k)\right|^{2}=\|D B \boldsymbol{c}\|_{2}^{2}
$$

where we have introduced the vector $\mathbf{c}(j)=c_{j}$ for $j \in \mathbb{Z}$, the matrix $B(k, j)=g^{\prime}(k, j), k \in\{-(N-$ $1), \ldots, N-1\}, j \in \mathbb{Z}$ and the $2 N-1$ by $2 N-1$ diagonal matrix $D$ with $D(k, k)=\sqrt{N-|k|}$ for $k \in\{-(N-1), \ldots, N-1\}$. Denoting the matrix $A(k, j)=g(k, j)$, we can represent the optimization problem as follows:

$$
\{\mathbf{c}\}=\operatorname{argmin}\|D B \mathbf{c}\|_{2}^{2} \quad \text { s.t. } \quad A \mathbf{c}=b .
$$

Here $\|\cdot\|_{2}$ denotes, as usual, the Euclidean norm. In applications the spectrum of the OFDM signal typically has to satisfy some specifi c decay requirements (a spectral mask) in order to avoid interference with other users operating in adjacent frequency bands. Often these decay requirements are enforced by an appropriate fi ltering of the continuous-time OFDM signal before transmission. Unfortunately this filtering can cause a loss of orthogonality of the subcarriers and thus lead to ICI. To avoid this unpleasant effect we can a priori design the OFDM pulse such that it satisfi es a prescribed spectral mask.

We can write these frequency decay requirements for the pulse $P(w)$ as $|P(w)| \leq S(w)$, where $S$ is a non-negative unimodal function representing some spectral mask. Taking into account this spectral decay constraint we arrive at the following constrained optimization problem:

$$
\{\mathbf{c}\}=\operatorname{argmin}\|D B \mathbf{c}\|_{2}^{2} \quad \text { s.t. } \quad A \mathbf{c}=b, \quad|P(w)| \leq S(w)
$$

It is straightforward to see that $\psi(c)=\|D B c\|_{2}^{2}$ is a convex function [11] and that the domain $\{\boldsymbol{c} \mid A \boldsymbol{c}=$ $\left.b,\left|P_{\boldsymbol{c}}(w)\right| \leq S(w)\right\}$ is a convex set. For the latter claim, we let $\Gamma=\left\{\boldsymbol{c}|A \boldsymbol{c}=b,| P_{\boldsymbol{c}}(w) \mid \leq S(w)\right\}$, and $\boldsymbol{c}_{1}, \boldsymbol{c}_{2} \in \Gamma$. For any $\delta \in(0,1)$, we consider vector $\boldsymbol{c}_{3}=\delta \boldsymbol{c}_{1}+(1-\delta) \boldsymbol{c}_{2}$. It is clear that $A \boldsymbol{c}_{3}=\delta A \boldsymbol{c}_{1}+(1-$ $\delta) A \boldsymbol{c}_{2}=b$. Further $P_{\boldsymbol{c}_{3}}(w)=\delta P_{\boldsymbol{c}_{1}}(w)+(1-\delta) P_{\boldsymbol{c}_{2}}(w)$, so $\left|P_{\boldsymbol{c}_{3}}(w)\right| \leq \delta\left|P_{\boldsymbol{c}_{1}}(w)\right|+(1-\delta)\left|P_{\boldsymbol{c}_{2}}(w)\right| \leq S(w)$. Thus we have $c_{3} \in \Gamma$, which shows the claim. Therefore we can find the global minimum by solving this convex optimization problem (15).

Since (15) describes an infi nite dimensional optimization problem, it is not directly accessible to a numerical solution. Hence in the next section we will propose an approach how to solve (15) numerically.

\section{NUMERICAL SOLUTION OF PULSE OPTIMIZATION PROBLEM}

In order to solve (15) numerically, we need to deal with a fi nite model. Therefore we fi rst truncate our pulse as $P_{n}(w)$ with expression

$$
P_{n}(w)=\sum_{j=-n}^{n} c_{j} \frac{\sin \left(\pi T(1+\alpha)\left(w-\frac{j}{(1+\alpha) T}\right)\right)}{(1+\alpha) T \pi w-\pi j} .
$$


Furthermore, we introduce the corresponding vector $\mathbf{c}^{(n)}$, the $2 N-1$ by $2 n+1$ matrices $B^{(n)}$ and $A^{(n)}$, where $\mathbf{c}^{(n)}(j)=c_{j}, B^{(n)}(k, j)=g^{\prime}(k, j)$ and $A^{(n)}(k, j)=g(k, j)$ for $j=-n, \ldots n$ and $k=$ $-(N-1), \ldots, N-1$. Hence the optimization problem becomes:

$$
\left\{\mathbf{c}^{o p t}\right\}=\operatorname{argmin}\left\|D B^{(n)} \mathbf{c}^{(n)}\right\|_{2}^{2} \quad \text { s.t. } \quad A^{(n)} \mathbf{c}^{(n)}=b, \quad\left|P_{n}(w)\right| \leq S(w) .
$$

It is easy to see that a necessary condition to satisfy $A^{(n)} \mathbf{c}^{(n)}=b$ is that $n>N-1$. In fact, we may have to let $n$ be quite larger than $N-1$ in order to satisfy both $A^{(n)} \mathbf{c}^{(n)}=b$ and $\left|P_{n}(w)\right| \leq S(w)$, which can increase the computational costs dramatically. On the other hand, considering (11) and the constraint in (17) we know $\left|c_{j}\right| \leq S\left(\frac{j}{1+\alpha}\right)$ which goes to zeros very fast especially for pulses whose Fourier transform decays fast. This observation suggests that a smaller $n$ might suffi ce to achieve an accurate result.

Suppose the pulse $H(w)$ satisfi es $|H(w)| \leq S(w)$ but does not satisfy (3). In this case we can make a re-correction process and let

$$
H_{1}(w):=H(w)+\sum_{k=-(N-1)}^{N-1}(b(k)-H(k)) Q(w-k),
$$

where the function $Q$ satisfi es

$$
Q(k)= \begin{cases}1 & k=0 \\ 0 & k \neq 0\end{cases}
$$

Literature offers many choices for $Q$, such as the rectangular pulse, a raised cosine pulse and other Nyquist Pulses. Thus $H_{1}(w)$ is the new pulse which satisfi es (3). This observation is the motivation to set up the truncated optimization problem by pursuing a pulse with expression:

$$
P_{n}(w)=\sum_{j=-n}^{n} c_{j} \frac{\sin \left(\pi T(1+\alpha)\left(w-\frac{j}{(1+\alpha) T}\right)\right)}{(1+\alpha) T \pi w-\pi j}+\sum_{k=-(N-1)}^{N-1} \bar{c}_{k} Q(w-k)
$$

and $\bar{c}_{k}=b(k)-H_{2}(k)$, where $H_{2}(w)=\sum_{j=-n}^{n} c_{j} \frac{\sin \left(\pi T(1+\alpha)\left(w-\frac{j}{(1+\alpha) T}\right)\right)}{(1+\alpha) T \pi w-\pi j}$. Hence, by construction for any $n, P_{n}(w)$ satisfi es (3). Then the TICI of pulse $P_{n}(w)$ is

$$
\sum_{k=-(N-1), k \neq 0}^{N-1}(N-|k|)\left|P_{n}^{\prime}(k)\right|^{2}=\left\|D\left(Q^{\prime}+B^{(n)} \mathbf{c}^{(n)}\right)\right\|_{2}^{2}
$$

where $B^{(n)}(k, j)=\frac{\partial^{2} P_{n}}{\partial w \partial c_{j}}(k)$ and $Q^{\prime}(k)=\frac{\partial Q}{\partial w}(k)$. Therefore, the truncated optimization problem becomes

$$
\left\{\mathbf{c}^{(n)}\right\}=\operatorname{argmin}\left\|D\left(Q^{\prime}+B^{(n)} \mathbf{c}^{(n)}\right)\right\|_{2}^{2} \quad \text { s.t. } \quad\left|P_{n}(w)\right| \leq S(w),
$$

where $P_{n}(w)$ is given in (20). In problems (22) and (20) $n$ can be any positive integer and $Q(w)$ can be a rectangular pulse, a raised cosine pulse and other Nyquist Pulses.

It remains to clarify the relation between the solution of the infi nite-dimensional optimization problem (15) and the truncated, modifi ed problem (22) which is accessible to numerical methods. We have the following theoretical results. 
Lemma 4.1: If $S_{1}(w)$ and $S_{2}(w)$ are two different constraints in (22), $S_{1}(w) \leq S_{2}(w)$ holds for all $w$, then the minimum of (22) with constraint $S_{1}(w)$ is no less than the minimum of (22) with constraint $S_{2}(w)$.

Proof: Suppose the solution for (22) with constraint $S_{1}(w)$ is $\mathbf{c}_{1}$ and the minimum is $d_{1}$, the corresponding pulse $P_{\mathbf{c}_{1}}$ satisfi es $\left|P_{\mathbf{c}_{1}}(w)\right| \leq S_{1}(w) \leq S_{2}(w)$, which means $\mathbf{c}_{1}$ is in the feasibility region of problem (22) with constraint $S_{2}$, and the minimum of (22) with constraint $S_{2}(w)$ is less than or equal to $d_{1}$.

The following observation is obvious.

Lemma 4.2: In the optimization problem (17), when $n$ increases, the minimum of (17) decreases.

Lemma 4.3: If $S(w) \in L^{2}(\mathbb{R})$ and optimization problem (15) has a solution, then when $n$ goes to $\infty$, the minimum of problem (17) has a limit which is bounded by the minimum of problem (15).

Proof: By Lemma 4.2, when $n$ increase, the corresponding minimum of problem (17) is a decreasing sequence. For any fi xed $n$, the minimum of (17) is no less than the minimum of problem (15). Thus we have the above result.

Based on these findings we fi nally arrive at the following result:

Theorem 4.4: If $S(w) \in L^{2}(\mathbb{R})$ and optimization problem (15) has a solution, when $n$ goes to $\infty$, the minimum of problem (22) has a limit which is bounded by the minimum of problem (15).

Proof: When $n$ increases, the feasibility region of problem (22) increases, thus the minimum of problem (22) decreases. Since the pulse in problem (22) satisfi es (3) and the constraint $\left|P_{n}(w)\right| \leq S(w)$, and its inverse Fourier transform is a time limited function in domain $\left[\frac{(-1-\alpha) T}{2}, \frac{(1+\alpha) T}{2}\right]$, the corresponding coeffi cient of pulse $P_{n}(w)$ is inside the feasibility region of problem (15). Therefore the minimum of problem (22) is no less than the minimum of problem (15), which proves the theorem.

Usually, we can get a very good result by solving (22) even when $n$ is small. In the next section, we will show the simulations results when $Q(w)$ is the raised cosine pulse.

\section{Simulations AND COMPARisons}

In this section, we compare our optimal pulses with the RC pulse, the BTRC pulse and the POLY pulse. These pulses have different spectral decays. Thus in order to make the comparisons fair, we should use different decay requirements when comparing with different pulses. To make this precise, we give the following defi nitions, which will be used to characterize the spectral decay of the various OFDM pulses.

Definition 5.1: For a given pulse $P(w)$, if the function $S(\omega)$ is unimodal and satisfi es $|P(w)| \leq S(w)$, we say that $S(\omega)$ is an upper bound for the pulse $P(w)$.

In this paper, we are interested in two kinds of upper bounds of a pulse, which appear most often in practice to describe spectral masks: (i) a piecewise step function, and (ii) a piecewise log-linear function (i.e., the logarithm of this function is a piecewise linear function).

Definition 5.2: Let $P(\omega)$ be a given pulse and let $\Gamma=\left\{\ldots, w_{-2}, w_{-1}, 0, w_{1}, w_{2}, \ldots\right\}$, denote a set of points where $w_{k}<w_{k+1}$ and $\left|w_{k}\right|$ goes to $\infty$ as $|k|$ goes to $\infty$. If the piecewise step function $S(w)$ is an upper bound for $P(w)$ in the sense of the defi nition above on each interval $\left[w_{k}, w_{k+1}\right]$, we say $S(w)$ is a piecewise step upper bound (PSUB) of $P(w)$. Furthermore, if for any $w_{k}<w_{m}, S(w)$ minimizes $\int_{w_{k}}^{w_{m}} S(w) d w$ in all these PSUB functions, we say $S(w)$ is the minimum piecewise step upper bound (MPSUB). Similarly we can defi ne minimum piecewise log-linear upper bound (MPLLUB). 
We know from the definitions above that for a given pulse the MPSUB (or MPLLUB) is unique, which clearly reflects the pulse decay. Suppose $S_{p}(w)$ is the MPSUB of the pulse $P(w)$ defined on $\Gamma=\left\{\ldots, w_{-2}, w_{-1}, 0, w_{1}, w_{2}, \ldots\right\}$. We use the function $S$ as the constraint in optimization problem (22) when we compare this pulse with our optimal pulse, where

$$
S(w)=\left\{\begin{array}{cc}
1 & w \in\left[-w_{1}, w_{1}\right] \\
S_{p}(w) & w \notin\left[-w_{1}, w_{1}\right] .
\end{array}\right.
$$

Thus for the RC, BTRC and POLY pulses, we solve (22) by using the corresponding MPSUB and get our optimal pulses which are labeled $\mathrm{OP}_{\mathrm{RC}}, \mathrm{OP}_{\mathrm{BTRC}}$, and $\mathrm{OP}_{\mathrm{POLY}}$ respectively. The following three pictures show the TICI performance for the above pulses for a 128-subcarrier OFDM system. We solve (22)

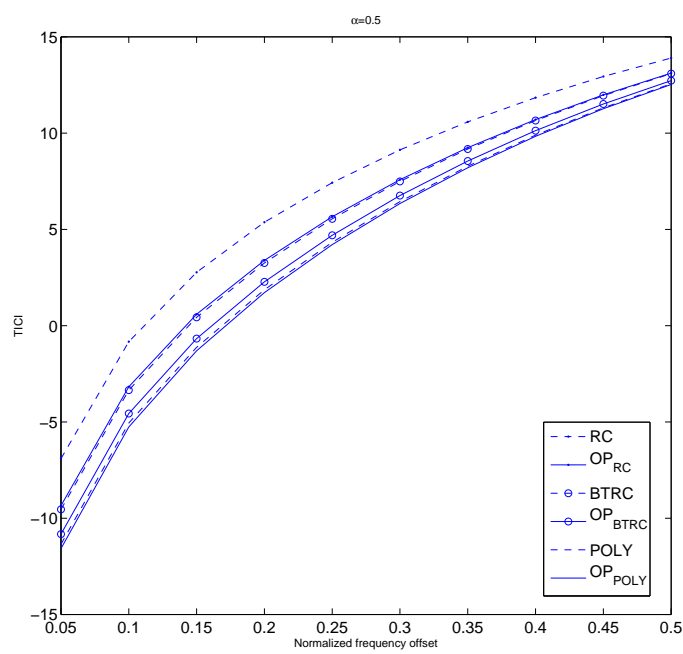

Fig. 1. The TICI of different OFDM pulse shapes for a 128-subcarrier OFDM system.

by using a sequential quadratic programming (SQP) method [12] which can be implemented by the Matlab function fminimax. We set $n=45$, which means we use 91 basis functions. Fig. 1 shows the numerical results when $\alpha=0.5$. In this case, at frequency offset 0.05 , the corresponding pulses can improve the TICI by $2.4 \mathrm{~dB}$ for the RC pulse, by $1.3 \mathrm{~dB}$ for the BTRC pulse and by $0.2 \mathrm{~dB}$ for the POLY pulse. For the comparison with the POLY pulse, we use the same parameters as in [4] (i.e., $\left.\left\{a_{2}=39, a_{3}=-99, a_{4}=85\right\}\right)$. In the case of the POLY pulse we see almost no improvement, the reason being that the POLY pulse has already been designed to be optimal with respect to the corresponding asymptotic decay rate. For the same $\alpha$, we have almost the same improvement for the TSIR criterion.

From the numerical results we fi nd that our optimal pulses have smaller TICI and higher TICI than the other pulses when the roll-off factor $\alpha<0.9$, independent of the frequency offset. We note from the standards 802.16, 802.11 and IS-54 that in practice one usually chooses $\alpha \leq 0.5$. Let us nevertheless briefly consider what happens when $\alpha \approx 1$. In this case our optimal pulses have slightly bigger TICI and 


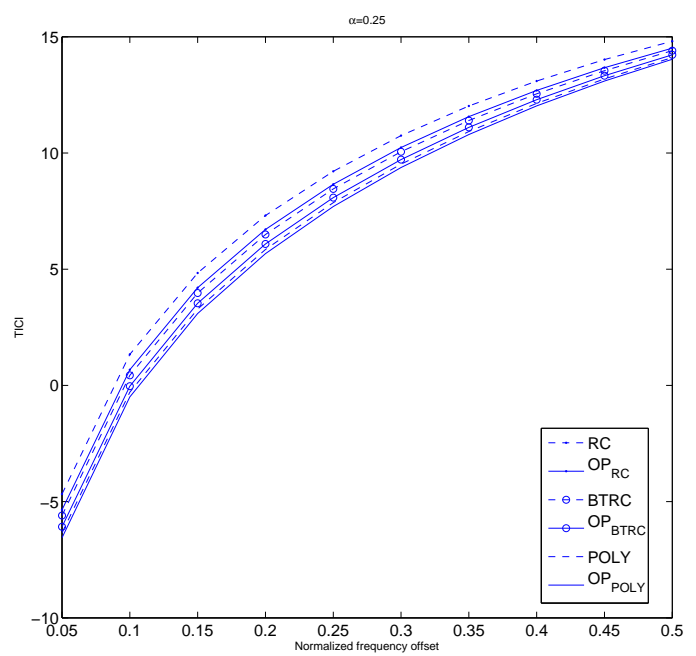

Fig. 2. The TICI of different OFDM pulse shapes for a 128-subcarrier OFDM system.

smaller TSIR than BTRC and POLY pulse when $\Delta f T$ is large, while when $\Delta f T<0.15$ the TSIR of our optimal pulses is much larger than the TICI of other pulses. When $\alpha=1$, our optimal pulses achieve the improvement of $13.7 \mathrm{~dB}, 8.7 \mathrm{~dB}$ and $5.1 \mathrm{~dB}$ for the RC pulse, BTRC pulse and POLY pulse (using the recommended parameters in [4]) respectively at frequency offset 0.05 . The different behavior with respect to $\Delta f T$ is not astonishing, since we initially have set up our optimization problem under the assumption that $\Delta f T$ is small.

From Lemma 4.1 we know that for a given pulse we can always fi nd another pulse with smaller TICI, but which has slower decay. Thus it is not surprising that the BTRC pulse and the POLY pulse have smaller TICI than the RC pulse, but this improvement comes at the cost of having a decay rate of only $\mathcal{O}\left(\frac{1}{w^{2}}\right)$ compared to a decay rate of $\mathcal{O}\left(\frac{1}{w^{3}}\right)$ for the RC pulse. However, as mentioned in Section III, in practice the Fourier transform of the continuous-time OFDM signal usually has to satisfy specific decay requirements in order to avoid interference with users operating in adjacent frequency bands. These decay requirements are often set forth in standard regulations in terms of spectral mask conditions. The OFDM pulses proposed in the literature-in particular those which have slower spectral decay than the raised cosine-may give rise to OFDM signals that do not meet those requirements. It is therefore desirable to be able to construct OFDM pulses that are guaranteed to meet decay requirements specifi ed by some prescribed spectral mask. The optimization problem in (15) (and its fi nite counterparts) includes such constraints.

In the following, we consider some typical examples for such spectral decay conditions. For purposes of reproducibility of research we give a detailed description of the decay requirements that we use (the reader who is not interested in these details may skip the following lines and just look at the spectral 


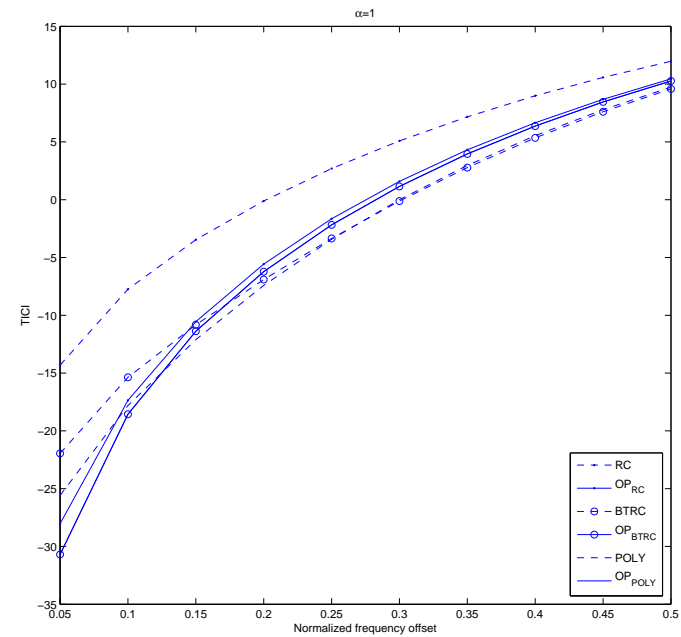

Fig. 3. The TICI of different OFDM pulse shapes for a 128-subcarrier OFDM system.

masks displayed in Fig. 4 and Fig. 5). We defi ne

$$
S(w)=\left\{\begin{array}{cc}
1 & w \in\left[-w_{1}, w_{1}\right], \\
\beta S_{p}(w) & w \notin\left[-w_{1}, w_{1}\right],
\end{array}\right.
$$

where $S_{p}(w)$ is the MPSUB or MPLLUB of the RC pulse.

Using the roll off factor $\alpha=0.5$ and $\beta=4$, we fi rst let $S_{p}(w)$ be the MPSUB of the RC pulse. The numerical results are shown in Fig. 4. In this case, at frequency offset 0.05 , our pulse achieves $3.25 \mathrm{~dB}$ improvement in TICI when compared with RC pulse. Then we let $S_{p}(w)$ be the MPLLUB of the RC pulse, the corresponding optimal pulse can achieve $3.7 \mathrm{~dB}$ improvement in TICI when compared with the RC pulse. The numerical results are shown in Fig. 5. These signifi cant improvements are made possible by optimally adapting the OFDM pulse to the prescribed spectral mask, while at the same time minimizing TICI.

In Table I, we compare the BER in the presence of time sampling errors computed according to [13]. For different roll-off factor $\alpha$, the optimal pulses have smaller BER than RC and BTRC pulses. The optimal pulses have smaller BER than POLY pulses at small time sampling errors, when the time sampling errors are large, the optimal pulses have a little larger BER since we set up the optimization problem by assuming the time sampling errors are small. Fig. 6 shows the fi gures of the time domain optimal pulses.

\section{CONCLUSION}

With increasing bandwidth and mobility wireless communication systems employing OFDM becomes increasingly sensible to Doppler effect and carrier frequency offset. It is thus important to have a flexible 
TABLE I

ISI ERROR PROBABILITY COMPARISONS FOR $N=2^{9}$ INTERFERING SYMBOLS

\begin{tabular}{c||c||cccc}
\hline$\alpha$ & pulse & $t / T= \pm 0.05$ & $t / T= \pm 0.1$ & $t / T= \pm 0.15$ & $t / T= \pm 0.2$ \\
\hline \hline \multirow{5}{*}{0.25} & RC & $8.219 \mathrm{e}-8$ & $2.818 \mathrm{e}-6$ & $7.153 \mathrm{e}-5$ & $9.746 \mathrm{e}-4$ \\
& $O P_{R C}$ & $6.497 \mathrm{e}-8$ & $1.705 \mathrm{e}-6$ & $3.810 \mathrm{e}-5$ & $5.234 \mathrm{e}-4$ \\
& BTRC & $5.812 \mathrm{e}-8$ & $1.298 \mathrm{e}-6$ & $2.622 \mathrm{e}-5$ & $3.568 \mathrm{e}-4$ \\
& $O P_{B T R C}$ & $5.141 \mathrm{e}-8$ & $1.069 \mathrm{e}-6$ & $2.141 \mathrm{e}-5$ & $2.916 \mathrm{e}-4$ \\
& POLY & $4.734 \mathrm{e}-8$ & $8.834 \mathrm{e}-7$ & $1.661 \mathrm{e}-5$ & $2.241 \mathrm{e}-4$ \\
& $O P_{P O L Y}$ & $4.566 \mathrm{e}-8$ & $8.816 \mathrm{e}-7$ & $1.748 \mathrm{e}-5$ & $2.393 \mathrm{e}-4$ \\
\hline \multirow{5}{*}{0.5} & RC & $3.972 \mathrm{e}-8$ & $5.489 \mathrm{e}-7$ & $8.174 \mathrm{e}-6$ & $1.022 \mathrm{e}-4$ \\
& $O P_{R C}$ & $2.612 \mathrm{e}-8$ & $2.512 \mathrm{e}-7$ & $3.5560 \mathrm{e}-6$ & $4.510 \mathrm{e}-5$ \\
& BTRC & $2.413 \mathrm{e}-8$ & $1.858 \mathrm{e}-7$ & $1.940 \mathrm{e}-6$ & $2.088 \mathrm{e}-5$ \\
& $O P_{B T R C}$ & $2.030 \mathrm{e}-8$ & $1.414 \mathrm{e}-7$ & $1.624 \mathrm{e}-6$ & $2.021 \mathrm{e}-5$ \\
& POLY & $2.057 \mathrm{e}-8$ & $1.354 \mathrm{e}-7$ & $1.372 \mathrm{e}-6$ & $1.520 \mathrm{e}-5$ \\
& $O P_{P O L Y}$ & $1.922 \mathrm{e}-8$ & $1.240 \mathrm{e}-7$ & $1.400 \mathrm{e}-6$ & $1.804 \mathrm{e}-5$ \\
\hline
\end{tabular}
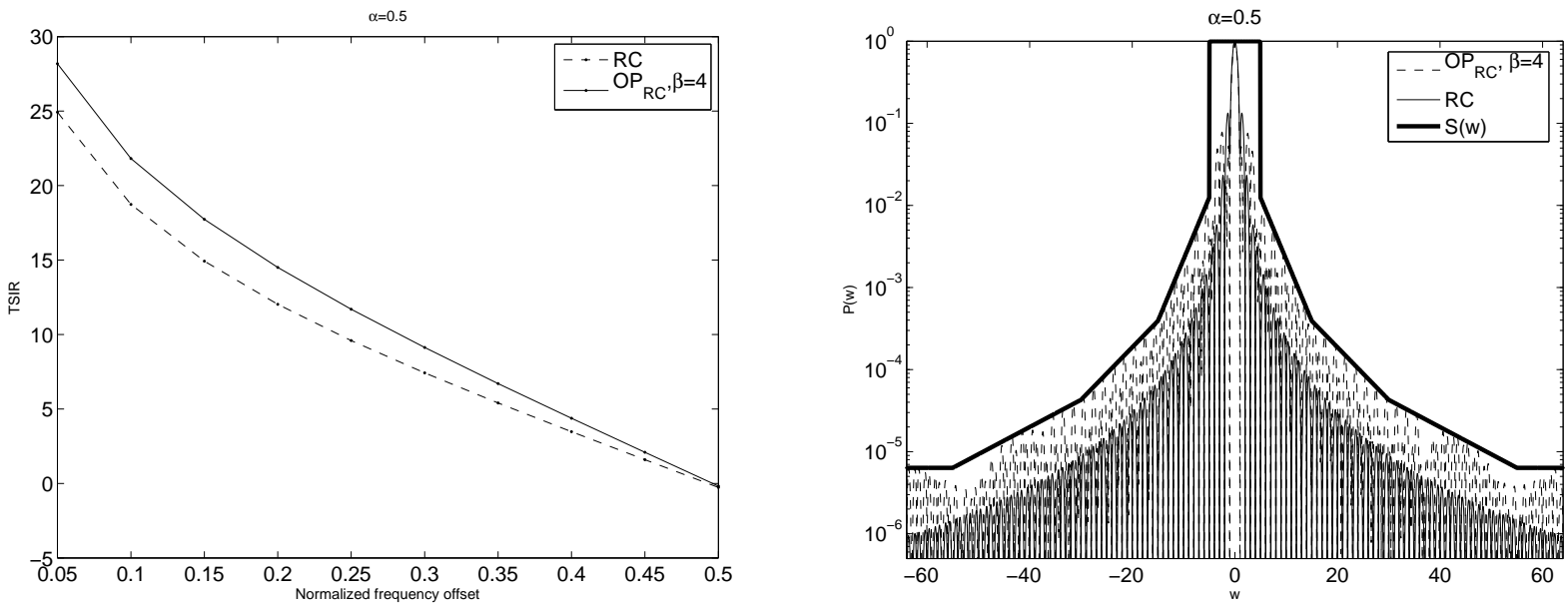

Fig. 4. Raised cosine and optimized pulse for a specifi c spectral mask.

framework at our disposal for the design of OFDM pulse shapes which can mitigate ICI. We have introduced a constrained optimization approach which yields OFDM pulses that outperform existing OFDM pulses with respect to ICI, and at the same time satisfy specifi c spectral mask requirements.

\section{REFERENCES}

[1] R. van Nee and R. Prasad, OFDM for Wireless Multimedia Communications, Artech House, Norwood, MA, 2000.

[2] N.S. Alagha and P. Kabal, "Generalized raised-cosine fi lters," IEEE Transaction on Communications, vol. 47, pp. 989-997, July,1999.

[3] Tan Peng and N.C. Beaulieu, "Reduced ICI in OFDM systems using the "better than" raised-cosine pulse," IEEE Communications Letters, vol. 8, pp. 135-137, MAR,2004.

[4] S. Chandan, P. Sandeep, and A.K. Chaturvedi, "A family of ISI-free polynomial pulses," IEEE Communications Letters, vol. 9, pp. 496-498, JUN,2005. 

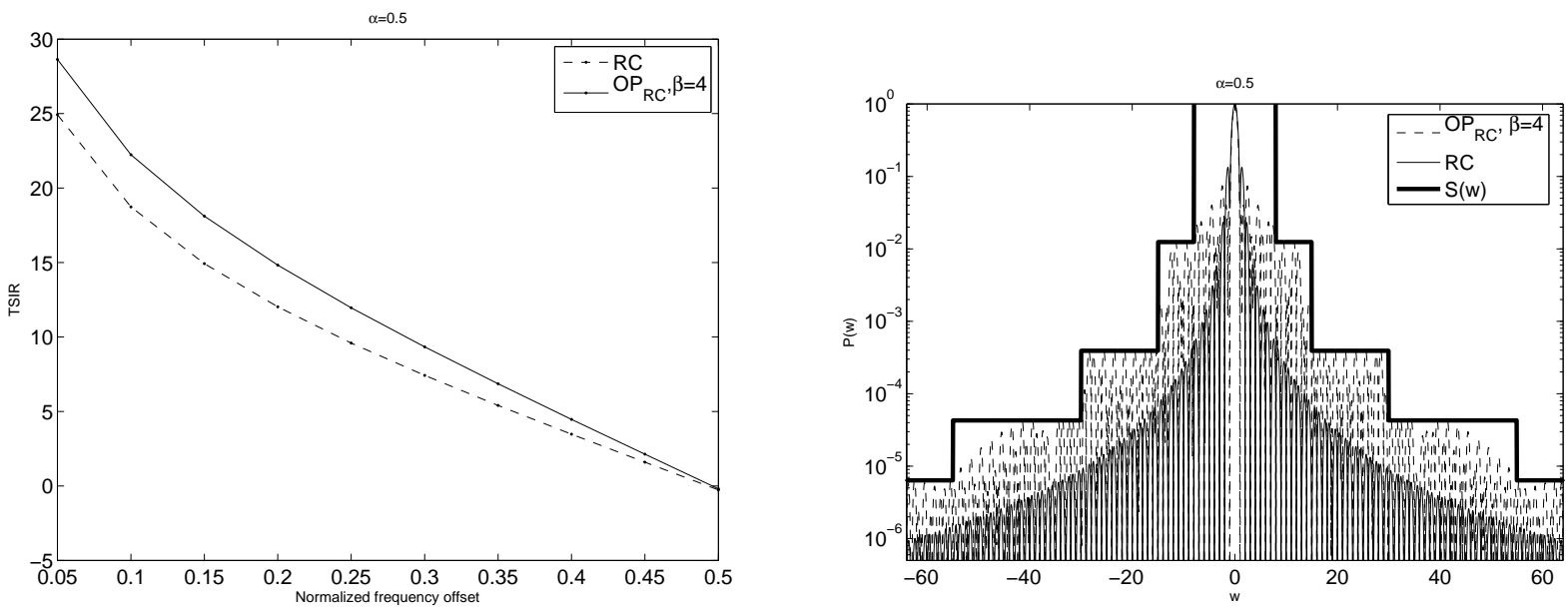

Fig. 5. Raised cosine and optimized pulse for another spectral mask.


Fig. 6. Optimal pulses for 128-subcarrier OFDM, using MPLLUB of the RC pulse as constraint.

[5] Assalini A. and Tonello A.M., 'Improved Nyquist pulses," IEEE Communications Letters, vol. 8, pp. 87-89, Feb,2004.

[6] R. Haas and J.C. Belfi ore, "A time-frequency well-localized pulse for multiple carrier transmission," Wireless Personal Communications, vol. 5, pp. 1-18, 1997.

[7] T. Strohmer and S. Beaver, 'Optimal OFDM system design for time-frequency dispersive channels," IEEE Trans. Comm., vol. 51, no. 7, pp. 1111-1122, 2003.

[8] D. Schafhuber, G. Matz, and F. Hlawatsch, 'Pulse-shaping OFDM/BFDM systems for time-varying channels: ISI/ICI analyis, optimal pulse design, and effi cient implementation," in Proc. IEEE PIMRC-02, Lisbon, Portugal, Sept. 2002, pp. $1012-1016$.

[9] Athanasios Papoulis, Signal Analysis, McGraw-Hill, Singapore, 1984.

[10] A. Beurling and P. Malliavin, "On the closure of characters and the zeros of entire functions," Acta Math., vol. 118, pp. 79-93, 1967.

[11] Stephen Boyd and Lieven Vandenberghe, Convex optimization, Cambridge University Press, Cambridge, 2004.

[12] R.K. Brayton, S.W. Director, G.D. Hachtel, and L.Vidigal, "A new algorithm for statistical circuit design based on quasi-newton 
methods and function splitting," IEEE Trans. Circuits and Systems, vol. CAS-26, pp. 784-794, Sept. 1979.

[13] Norman C.Beaulieu, "The evaluation of error probabilities for inter symbol and cochannel interference," IEEE Transcations on Communications, vol. 39, pp. 1740-1749, Dec 1991. 